\title{
Constraining effects of examples in a creative generation task
}

\author{
STEVEN M. SMITH, THOMAS B. WARD, and JAY S. SCHUMACHER \\ Texas A\&M University, College Station, Texas
}

\begin{abstract}
In three experiments we tested the conformity hypothesis-that subjects' ideas would conform to examples they had been shown-by using a creative generation paradigm in which subjects imagined and sketched new exemplars of experimenter-defined categories. Designs made by subjects who had first seen three examples of ideas were compared with those of control subjects, who received no examples. In all three experiments, the designs of subjects who had seen the examples were more likely to contain features of the examples. This conformity effect did not significantly decrease in Experiment 2, when a 23-min task was interpolated between viewing the examples and generating related ideas. The hypothesis that the observed conformity effects may have been caused by subjects' assumptions that they should try to generate ideas similar to the examples was refuted in Experiment 3; explicitly instructing subjects to create ideas that were very different from the examples did not decrease conformity to the examples, and instructing them to conform to the examples significantly increased conformity. The results show that recent experience can lead to unintentional conformity, constraining the generation of creative ideas.
\end{abstract}

Creative thinking produces new ideas and novel solutions to problems, and is therefore crucial for adapting to varied or changing environmental demands. Many special endeavors such as invention, theory or product development, and creative writing, as well as common activities such as extemporaneous conversation, depend upon creative thinking. Although creativity has often been studied as an individual difference factor, our approach is to examine the cognitive processes and structures that underlie creative thinking, particularly as they relate to the interactive stages of idea generation and exploration (Finke, Ward, \& Smith, 1992).

Finke et al. (1992) proposed a cognitive model of creative thinking called geneplore, a name that emphasizes the importance of generative and exploratory phases of the creative process. In the generative phase, one uses processes such as retrieval, analogical transfer, or mental transformation to construct representations of ideas that may take various forms such as visual patterns, verbal combinations, or mental models. These initial ideas, referred to as preinventive forms, ideally have properties such as novelty, meaningfulness, and emergent qualities. Exploratory processes can then be used to develop the initially generated ideas for specific purposes.

A category generation paradigm was used in the present experiments to study the initial stages of idea production.

This research was supported in part by NIMH Grant R01 MH4473001 to S.M.S. The authors wish to thank Jill Bily, Kindell Foley, and Laura Rodriguez for their help in collecting the experimental data, to Stephen Balfour and Jeffrey Brown for their help in scoring the data, and to R. E. Anderson and T. Helstrup for helpful comments on an earlier draft of this paper. Correspondence should be addressed to $S$. Smith, Department of Psychology, Texas A\&M University, College Station, Texas 77843 .
Subjects were asked to think of new and creative exemplars of specified categories: toys and creatures from an imaginary planet like Earth. In this task, subjects are assumed to begin by retrieving or constructing a representation of the specified category, be it in the form of features, exemplars, or prototypes. They then use that representation to produce new exemplars. Although this task may involve exploration as well as generation, its requirements stress that subjects should produce as many new ideas as possible in a limited time, therefore limiting the degree of exploration in which they are likely to engage.

In the present study, we were particularly concerned with the effects of prior experiences in the creative idea generation task. Although prior experiences can provide the expertise needed to fully explore and develop ideas into creative products, it may be that such experiences limit creative thinking when ideas are originally being generated. Because the representation of a category that is constructed on a given occasion can be influenced by recent events and the current context (e.g., Barsalou, 1987), it is possible that prior experiences could bias the production of new category exemplars. Do prior experiences always benefit creative thinking, or can they constrain creative idea generation by leading people to base their ideas on the details of those earlier experiences?

Benefits of prior experience are well documented in a variety of complex cognitive tasks, such as comprehension (Bransford, Barclay, \& Franks, 1972), analogical reasoning (e.g., Gick \& Holyoak, 1980), and problem solving (e.g., Ross, Ryan, \& Tenpenny, 1989). Facilitated performance can result from well-learned knowledge, including factual information, heuristics, and analogies. It can also result from recent experiences, such as when examples are given along with a task. 
Those who provide examples for educational purposes may intend them as hints that will help students reach known goal states, perhaps by suggesting transfer of relations, heuristics, or algorithms from the examples to the task at hand. Examples are commonly given in professional settings that require problem solving, such as architecture or engineering. In open-ended situations in which novel creations are sought to satisfy some need, examples may be given to demonstrate useful past approaches, or to show the pitfalls discovered by those engaged in prior related work. It seems obvious that examples are usually not intended to impede the generation process, but rather to enhance the flow of useful ideas.

On the other hand, there are situations in which prior experiences can have a damaging, counterproductive effect on the outcome of cognitive processing. If knowledge is applied inappropriately, or if ideas are unnecessarily constrained because of prior experiences, then performance could suffer or fail. Such negative transfer, in which the use of prior experience is counterproductive, has been referred to as cognitive fixation, which has been attributed to the same processes that cause interference effects in memory (e.g., Smith \& Blankenship, 1991).

There are a number of phenomena that demonstrate fixation in memory and problem solving. In the classic studies using the "water jar" problem (e.g., Luchins \& Luchins, 1959), subjects saw numerous problems prior to critical test problems. The problems leading up to the critical one could all be solved with the same strategy, a solution referred to as the set or Einstellung solution. On the critical test problem, the subjects typically tried to use the set solution, even when it was inappropriate. Thus, the set solution that is learned on early trials impedes or fixates the subject's search for a solution.

Smith and Blankenship $(1989,1991)$ manipulated fixating cues directly in order to examine the effects of recent experiences on a different problem-solving task. In an incidental task given prior to problem solving, subjects were presented with misleading distractors related to problem solutions. For example, the solution to the Remote Associates Test (RAT) problem " APPLE HOUSE FAMILY" is "tree," because the word tree can form a twoword phrase with each test word: APPLE-tree, treeHOUSE, and FaMILY-tree. Subjects who initially studied the distractor pairs APPLE-pie, HOUSE-warming, and FAMILY-secret on an incidental learning task performed poorly on the subsequent RAT problems. Such distractors reliably diminished problem-solving performance, as compared with conditions in which unrelated distractors or no distractors were presented to subjects. These experiments demonstrate counterproductive effects of recent memory, an example of fixation in problem solving.

Fixation can also block access to material in memory tasks. An example of this is the phenomenon of part-set cuing inhibition (e.g., Nickerson, 1984; Slamecka, 1968); recall of a critical list of items is worse if other items from the memory set are given as "cues" at the time of recall, as compared with a "no cues" test. Unintuitively, and in contradiction to many theories that encompass only beneficial effects of recall cues, the part-set cuing phenomenon demonstrates a case in which examples (part-list cues) can serve as impediments to the generation of targets in a memory set. A commonly accepted explanation of the phenomenon states that output interference from the provided list cues causes competition at recall, effectively blocking retrieval of other list items (e.g., Raaijmakers \& Shiffrin, 1981; Roediger \& Neely, 1982; Rundus, 1973). Thus, items that are given as cues constrain retrieval, impeding retrieval of noncue targets. This analysis suggests that a conventional memory mechanism, such as interference or response competition, may underlie various forms of fixation in both memory and problem solving.

Most fixation effects have been observed in tasks in which a single correct, or single best answer exists, and the emphasis has been on how subjects are prevented from converging on such answers. In contrast, creative thinking is not often assessed in this convergent, veridical way. Rather, it is usually judged on the basis of criteria such as novelty, originality, or flexibility. Thus, examining the nature of creative idea generation suggests another possible inhibiting effect of prior experiences. Those experiences may not only prevent ideas per se, but may also cause ideas to be less novel or original. In the present study, such constraining effects of prior experiences on creative idea generation will be referred to as conformity, because ideas generated may conform to the features of related exemplars that have been recently experienced. It may be that both the fixation that prevents correct solutions and the conformity that inhibits novelty have the same underlying cause.

Our focus in the present paper is on the extent to which conformity effects occur in creative generation tasks. Do prior experiences inhibit the number or range of ideas in more open-ended generation tasks for which many possible answers exist? When individuals develop imaginative new ideas, such as inventions, theories, or ideas for stories, to what extent are such creations constrained by the examples they are given? Do they become constrained by the properties of examples they see? Might they be more innovative with no presented examples to constrain imagination?

Research by Ward (in press) provides indirect evidence for the idea that examples may lead to conformity in a creative idea generation task. Ward's subjects imagined and drew creatures that might exist on other planets, with the results showing that the degree of innovation in the subjects' drawings was related to their reported approach to the task. The subjects who reported basing their creations on specific known exemplars of Earth species included fewer innovations than other subjects. This finding is consistent with the idea that subjects become constrained by the properties of their own self-generated examples. 
Although Ward's results are consistent with the idea that people become constrained by the properties of examples, they offer only indirect evidence. The fact that the subjects who reported retrieving exemplars produced less innovative creatures could simply reflect individual differences in creativity. Correlated tendencies in less creative people may lead them to perform generation tasks by retrieving specific exemplars, and also to produce less innovative creations. By this interpretation, there would be no necessary causal link between the availability of an example and conforming to its properties. To provide direct evidence for conformity to the properties of examples in a creative generation task, it is important to experimentally manipulate their availability by presenting examples to some subjects and not to others. If examples produce conformity, then those who receive them should be more likely to generate products that have properties in common with the examples.

The idea-generation task used in the present experiments, as in Ward's (in press) studies, required creative thinking because it involved generating novel entities with one's imagination. Subjects given these tasks created as many different new imaginary creatures as possible, as the instructions emphasized both novelty and quantity. If examples cause subjects to predominantly generate ideas that are similar to the given examples, this would be considered counterproductive in terms of creativity, because it would constrain the novelty and the range of ideas generated in the task. In the present studies, it was predicted that the provided examples of ideas would constrain creative generation processes by causing the subjects' ideas to conform to features of the examples.

The present studies focused on several aspects of conformity effects in creative generation. The first study demonstrated that merely presenting individuals with examples in a creative generation task would bias their creations to contain the shared properties of those examples. In the second study, we examined whether or not such a biasing effect would be reduced by interposing a filled delay between the presentation of the examples and the performance of the generation task. In the final experiment, we tested whether the conformity effects were based on the subjects' deliberate attempts to create instances that contained the properties of the examples, or an unintended result of remembering those examples.

\section{EXPERIMENT 1}

College students generated ideas for as many different toys or imaginary creatures as they could in $20 \mathrm{~min}$. Existing toys and creatures were not allowed. It was hypothesized that if the subjects were shown examples that all had particular attributes in common, then a higher proportion of their creative designs would contain those attributes, compared with a control group that saw no examples prior to the generation task. This hypothetical result, that features of ideas generated by subjects tend to duplicate the features of examples, will be referred to as a conformity effect. Such an effect would be counterproductive for creative thinking in the present experiments, because the task was to generate as many new and different ideas as possible; ideas that conform to previously seen examples are less novel than those that do not conform.

\section{Method}

Subjects. The 94 participants were volunteers from introductory psychology classes who fulfilled a class requirement by participating in the experiment. The subjects in all three experiments were recruited by posting sign-up sheets for group sessions to be conducted at various times. The volunteers were free to enroll for any session of many experiments, including the present three experiments. Because equal numbers of subjects did not enroll for every session, there were unequal numbers of subjects in the treatment groups.

Experimental sessions were conducted in a large auditorium. The subjects were seated in every other seat in every other row so that they could not view the others' ideas. Materials corresponding to the two main treatment conditions, examples and control, were divided randomly among the subjects in each session.

Design and Procedure. For each task, creature generation and toy generation, the subjects were asked to sketch and label as many novel ideas as possible within the time allotted. In the creaturegeneration task, the subjects were given the following instructions: "Imagine a planet just like Earth existing somewhere in the universe. It is currently uninhabited. Your task is to design new creatures to inhabit the planet. Within the allotted 20 minutes draw as many new and different creatures of your own creative design as you are able. Duplication of creatures now extinct or living on the planet Earth is not permitted. Provide both a side view and a front view of each creature. After completing the drawing of a creature: (1) label each part of the creature, (2) briefly describe and explain the creature, and (3) continue to the next page and design a new creature."

In the toy-generation task, the subjects were told, "Imagine that you are employed by a toy company that is in need of new ideas for toys. Your task is to design some new toys for the company. Within the allotted 20 minutes draw as many new and different toys of your own creative design as you are able. Duplication of toys that currently exist or have already existed is not permitted. After completing the drawing of a toy: (1) label each part, (2) briefly describe and explain the toy, and (3) continue to the next page and draw a new toy."

For $90 \mathrm{sec}$, just prior to the first generation task, half of the groups were shown example designs (examples condition), and the other half were shown no examples (control condition). Each example was given on a separate page. The three pages of examples could all be seen for the entire 90 - sec viewing period. After the $90 \mathrm{sec}$, the examples were removed from sight and could not be viewed again during the experiment. The subjects in the control condition were asked to sit quietly during the $90 \mathrm{sec}$ and await further instructions.

After studying the three examples and removing them from view (or after no examples), the subjects spent 20 min drawing and labeling their creatures or toys. They were provided with many response pages for each task. Each response page for the creatures task had two spaces, one for each of the two drawings of a single design. The two spaces were labeled "front view" and "side view" for the creature-generation task. The reason for the two views was to later help scorers to determine whether a creature had four legs and/or a tail. Only one view of each toy was requested. One page was used for each design. 
Three examples for the second generation task were then shown for $90 \mathrm{sec}$ and removed (or no examples were shown), followed by a 20 -min session in which the subject generated ideas for creatures or toys, whichever they had not generated in the first task.

Conformity (examples vs. control) was manipulated between subjects in all experiments. Task order was counterbalanced; half of the groups generated first toys, then creatures, and the other half generated first creatures, then toys.

Materials. Sample designs for the toy and creature tasks were created by the authors. The three examples for each task had three attributes in common: each toy example had a ball as part of the design, involved a high level of physical activity, and used electronic devices. The sample creatures all had four legs, two antennae, and a tail. Each example was shown on a single page, which included two views of a creature or one view of a toy and labels of the parts of the drawing. The toy and creature examples are shown in Figures 1 and 2.

\section{Results}

The generated designs were scored by the third author in terms of the critical features of the examples: a ball, high level of physical activity, and electronics for the toys, and antennae, four legs, and a tail for the creatures. To obtain an interrater reliability measure, a second rater scored the designs independently. The overall interrater reliability was .84 . Interrater reliability for the toy designs was .78, and for the creature designs it was .90 . The reported results were based upon the scoring done by the first rater. Most of the features in question were
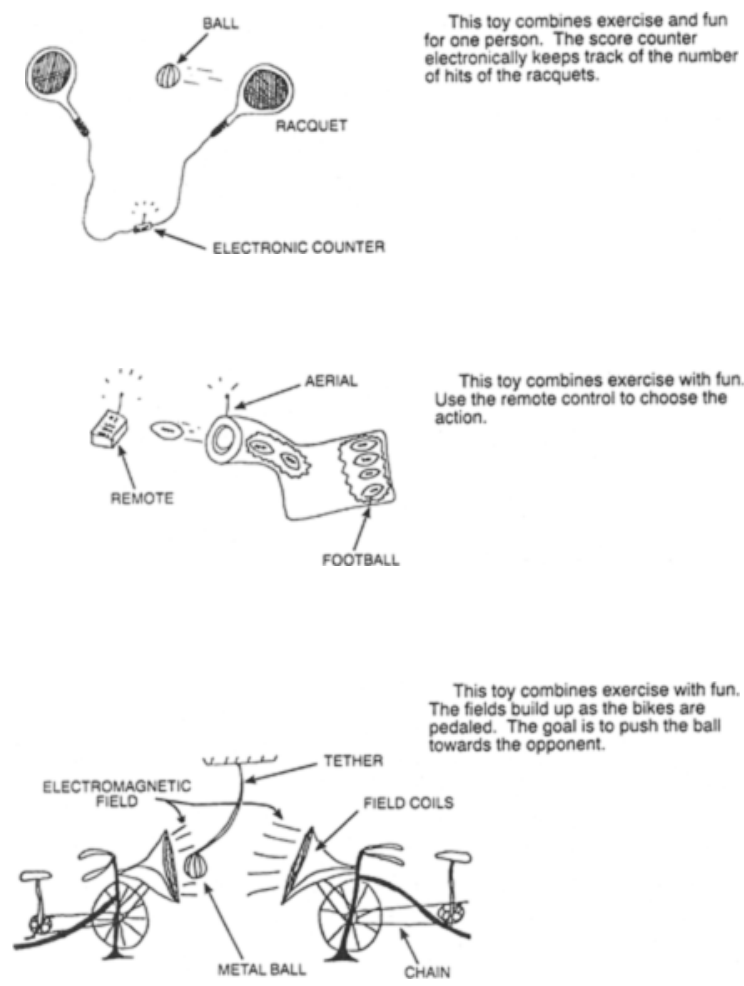

Figure 1. Example toys, along with their labels and descriptions, that were provided to examples subjects in Experiments 1 and 2. Each example used a ball, involved a high level of physical activity, and used electronics.

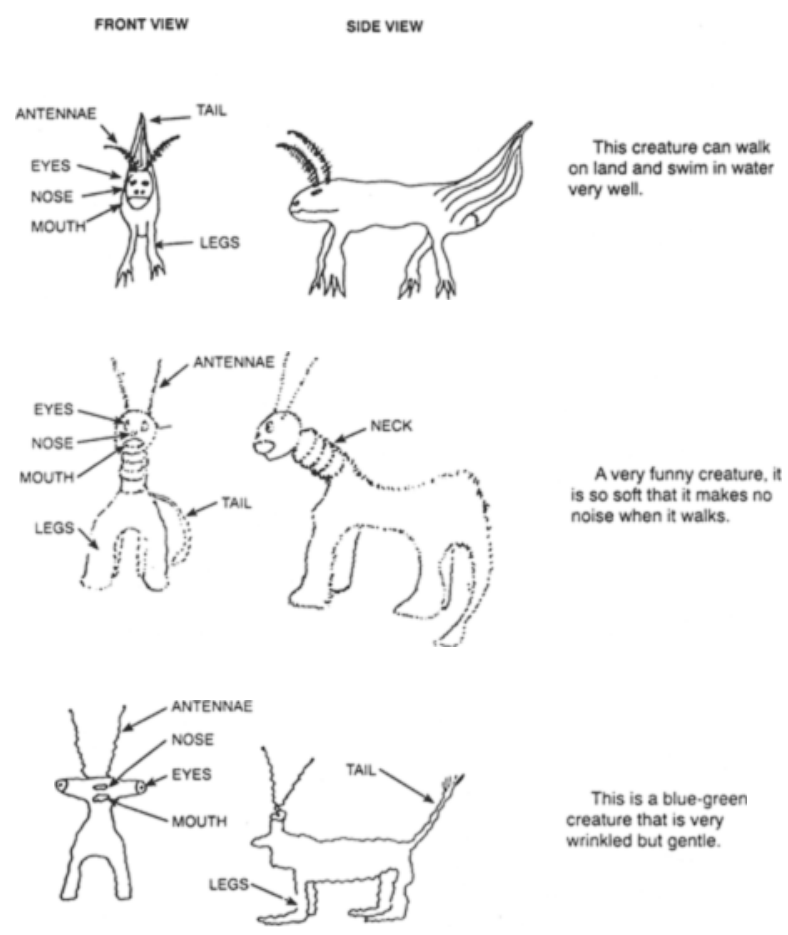

Figure 2. Example creatures, along with their labels and descriptions, that were provided to examples subjects in all three experiments. Each example had four legs, antennae, and a tail.

clearly labeled or described by the subjects, as they had been instructed. Unlabeled features of creature designs, such as four legs or tail, were usually easy to identify because of the two views, front and side, drawn for each creature. Features such as a ball, a tail, or antennae were scored as such, regardless of their relative sizes or proportions. A significance level of $p<.05$ was used in all reported comparisons in all three experiments.

Toys. Five separate one-way analyses of variance (ANOVAs) were computed for the toy designs, each testing the effect of conformity (examples vs. control). The dependent measures used were number of toy designs generated, proportion of generated toy designs that used a ball, proportion that involved a high level of physical activity, proportion using electronics, and overall conformity (the average of the three proportions). The proportions were calculated for each subject; the means for these measures are shown in Table 1. The same mean number of toy designs (Table 1), roughly three per subject, was created in the two treatment conditions $[F(1,92)=.11$, $\left.M S_{\mathrm{e}}=1.45\right]$.

Two of the three critical attributes were significantly more common for the examples group than for the control group (Table 1)-the proportion using a ball $[F(1,92)$ $\left.=10.59, M S_{\mathrm{e}}=.09\right]$ and the proportion involving a high level of physical activity $\left[F(1,92)=5.97, M S_{\mathrm{e}}=.10\right]$. Although numerically higher for the examples group, the proportion using electronics did not significantly differ for the two treatment groups $\left[F(1,92)=.19, M S_{\mathrm{e}}=.25\right]$. 
Table 1

Mean Number of Ideas Generated, With Standard Deviations, Proportions of Generated Ideas With Features of Examples, and Overall Conformity as a Function of the Examples Given in Experiment 1

\begin{tabular}{|c|c|c|c|c|}
\hline & \multicolumn{4}{|c|}{ Condition } \\
\hline & \multicolumn{2}{|c|}{ Control } & \multicolumn{2}{|c|}{ Examples } \\
\hline & $M$ & $S D$ & $M$ & $S D$ \\
\hline \multicolumn{5}{|c|}{ Toys } \\
\hline No. of toys & 2.82 & 1.06 & 2.90 & 1.31 \\
\hline Ball & .11 & .23 & .31 & .35 \\
\hline Physical & .23 & .28 & .39 & .34 \\
\hline Electronic & .30 & .64 & .35 & .33 \\
\hline Overall conformity & .22 & .23 & .35 & .23 \\
\hline \multicolumn{5}{|c|}{ Creatures } \\
\hline No. of creatures & 2.95 & 1.18 & 3.08 & .94 \\
\hline Antennae & .05 & .18 & .17 & .24 \\
\hline Tail & .15 & .28 & .37 & .30 \\
\hline Four legs & .14 & .24 & .19 & .23 \\
\hline Overall conformity & .11 & .18 & .24 & .17 \\
\hline$n^{*}$ & \multicolumn{2}{|c|}{44} & \multicolumn{2}{|c|}{50} \\
\hline
\end{tabular}

*Number of subjects for each group.

Overall conformity to the examples (the mean of the three proportions) was significantly higher for the examples group $\left[F(1,92)=8.13, M S_{\mathrm{e}}=.05\right]$, indicating a conformity effect.

Creatures. Five one-way ANOVAs were computed for creature designs by using the number of creatures generated, proportion of generated creatures with antennae, proportion with four legs, proportion with a tail, and overall conformity to the creature examples (the average of the three proportions) as dependent measures. Each ANOVA tested the effect of conformity (examples vs. control). The same mean number of creatures (Table 1), about three per subject, was created in both conditions $[F(1,92)=.33$, $\left.M S_{\mathrm{e}}=1.13\right]$.

Two of the three critical attributes were found more frequently in the examples group than in the control group (Table 1)-the proportion with antennae $[F(1,92)=7.24$, $\left.M S_{\mathrm{e}}=.05\right]$ and the proportion with a tail $[F(1,92)=$ 13.17, $M S_{\mathrm{e}}=.09$ ]. The proportion of generated creature designs with four legs did not significantly differ between the two treatment groups $\left[F(1,92)=1.07, M S_{\mathrm{e}}=.05\right]$, although it was somewhat higher for the examples group. Overall conformity to the creature examples (the mean of the three proportions) was significantly greater for the examples group $\left[F(1,92)=12.50, M S_{\mathrm{e}}=.03\right]$, again indicating a conformity effect.

\section{Discussion}

Conformity effects were found in the analyses of both toy and creature designs. Creative designs were constrained when the examples were shown prior to the generation task.

\section{EXPERIMENT 2}

In Experiment 2, we tested whether conformity effects in creative idea generation diminish over time. The method in Experiment 2 essentially replicated that used in Experiment 1 , except that all the examples, including both toys and creatures, were presented at the beginning of the experiment, rather than immediately before the most relevant generation task. Thus, some examples were studied immediately before a task, and others were studied $23 \mathrm{~min}$ before the relevant task. It was predicted that a conformity effect would be observed, and that the effect would be stronger when examples were presented immediately prior to the related tasks, rather than delayed.

\section{Method}

Subjects. The 91 participants were volunteers from introductory psychology classes who fulfilled part of a class requirement by participating in the experiment.

Design, Procedure, and Materials. The design, procedure, and materials for Experiment 2 were identical to those used in Experiment 1, with one exception. In Experiment 1, each set of examples was shown for $90 \mathrm{sec}$ immediately preceding the related task, but in Experiment 2, both sets of examples were shown prior to the first generation task. This means that the examples immediately preceded the first generation task (the immediate condition), but preceded the second task (the delayed condition) by $23 \mathrm{~min}$. Examples in the immediate condition were always shown as the second set of examples, ensuring that the task related to that set would be tested immediately after the examples had been shown. The order of the two tasks (creature and toy generation) was counterbalanced, as in Experiment 1.

\section{Results}

The generated designs for toys and creatures in Experiment 2 were scored the same way as described for Experiment 1. Each ANOVA computed for toys and creatures used a $2 \times 2$ (conformity $\times$ order/delay) design. Conformity was either examples or control, and order/delay was either first/immediate or second/delayed. That is, in the examples condition, the first task immediately followed the examples, and the second task was delayed from its examples. In the control condition, the terms immediate and delayed are irrelevant, because there were no examples given, so the terms first and second will be used for this condition.

Toys. Five separate ANOVAs were computed for the toy designs. The dependent measures used were the same as those used in Experiment 1. The means for these measures are shown in Table 2.

The same mean number of toy designs (Table 2) was found in both the examples and control conditions $[F(1,87)$ $\left.=.22, M S_{\mathrm{e}}=1.99\right]$. There was an effect of order; more toy designs were generated when toys were done first than when they were second $\left[F(1,87)=6.13, M S_{\mathrm{e}}=1.99\right]$.

Numerically, the proportions of toy designs containing critical features were all greater in the examples than control conditions, regardless of delay conditions (Table 2). For individual features, the effect was significant for the electronics attribute $\left[F(1,87)=7.06, M S_{\mathrm{e}}=.08\right]$, was not significant for the ball attribute $[F(1,87)=1.48$, $\left.M S_{\mathrm{e}}=.08\right]$, and was marginally significant for the physical activity attribute $\left[F(1,87)=3.55, M S_{\mathrm{e}}=.08, p=\right.$ .06]. Overall toy conformity showed the predicted significant constraining effect of examples $[F(1,87)=7.45$, $\left.M S_{\mathrm{e}}=.04\right]$. 
Table 2

Mean Number of Ideas Generated With Standard Deviations, Proportions of Generated Toys With Features of Examples, and Overall Conformity as a Function of the Examples Given and Delay/Order of Testing in Experiment 2

\begin{tabular}{|c|c|c|c|c|c|c|c|c|}
\hline & \multicolumn{8}{|c|}{ Condition } \\
\hline & \multicolumn{4}{|c|}{ Control } & \multicolumn{4}{|c|}{ Examples } \\
\hline & \multicolumn{8}{|c|}{ Delay/Order of Testing } \\
\hline & \multicolumn{2}{|c|}{ First } & \multicolumn{2}{|c|}{ Second } & \multicolumn{2}{|c|}{ Immediate/First } & \multicolumn{2}{|c|}{ Delayed/Second } \\
\hline & $M$ & $S D$ & $M$ & $S D$ & $M$ & $S D$ & $M$ & $S D$ \\
\hline No. of toys & 3.93 & 1.54 & 2.55 & 1.37 & 3.55 & 1.30 & 3.25 & 1.43 \\
\hline Ball & .15 & .24 & .21 & .31 & .24 & .26 & .28 & .30 \\
\hline Physical & .20 & .23 & .18 & .30 & .40 & .30 & .25 & .29 \\
\hline Electronic & .28 & .23 & .09 & .16 & .30 & .26 & .44 & .34 \\
\hline Overall conformity & .21 & .15 & .16 & .15 & .31 & .22 & .32 & .22 \\
\hline$n^{*}$ & \multicolumn{2}{|c|}{11} & \multicolumn{2}{|c|}{14} & \multicolumn{2}{|c|}{44} & \multicolumn{2}{|c|}{22} \\
\hline
\end{tabular}

There was no effect of order/delay on any of the measures. Order interacted with conformity only for the electronics attribute $\left[F(1,87)=5.66, M S_{\mathrm{e}}=.08\right]$, indicating a greater effect of the examples when there was a delay between the presentation of the toy examples and the generation of toy designs. Order and conformity did not interact for the overall toy conformity measure $[F(1,87)=.36$, $\left.M S_{\mathrm{e}}=.04\right]$.

Creatures. The results of the creature designs are shown in Table 3. On the number of creatures generated, there were no effects of conformity $\left[F(1,87)=.46, M S_{\mathrm{e}}=\right.$ $1.80]$, of order/delay $\left[F(1,87)=.35, M S_{\mathrm{e}}=1.80\right]$, or of the conformity $\times$ order/delay interaction $[F(1,87)=$ $\left..11, M S_{\mathrm{e}}=1.80\right]$.

An effect of conformity was found for proportion of creatures with antennae $\left[F(1,87)=9.11, M S_{\mathrm{e}}=.11\right]$ and proportion with four legs $\left[F(1,87)=10.92, M S_{\mathrm{e}}=.07\right]$, and the effect was marginal for proportion with a tail $\left[F(1,87)=3.51, M S_{\mathrm{e}}=.09, p=.06\right]$. Overall conformity of creatures to the examples was significantly affected by conformity $\left[F(1,87)=12.53, M S_{\mathrm{e}}=.05\right]$, as predicted.
The effect of order/delay on overall conformity of creatures to the examples was significant $[F(1,87)=4.61$, $\left.M S_{\mathrm{e}}=.05\right]$, indicating that there was greater overall conformity to the creature examples when the creatures were designed first, rather than second. This pattern was found, however, for both the examples and control groups; no conformity $\times$ order/delay interaction was found $[F(1,87)$ $\left.=.45, M S_{\mathrm{e}}=.05\right]$. This means that the lower "conformity" to the examples in the delayed conditions appears to have been due to an order effect, and should not be interpreted as a decline in example-induced conformity.

\section{Discussion}

The primary prediction was again supported by the results, replicating the major finding of Experiment 1. More of the designs of the examples group contained features of the examples, as compared with the control group. This finding was true for both creature and toy designs.

A delay of $23 \mathrm{~min}$ between showing the examples to the subjects and testing the relevant task did not significantly diminish the constraining effects of examples in the creative generation task, although there was a trend

Table 3

Mean Number of Ideas Generated With Standard Deviations, Proportions of Generated Creatures With Features of Examples, and Overall Conformity as a Function of the Examples Given and Delay/Order of Testing in Experiment 2

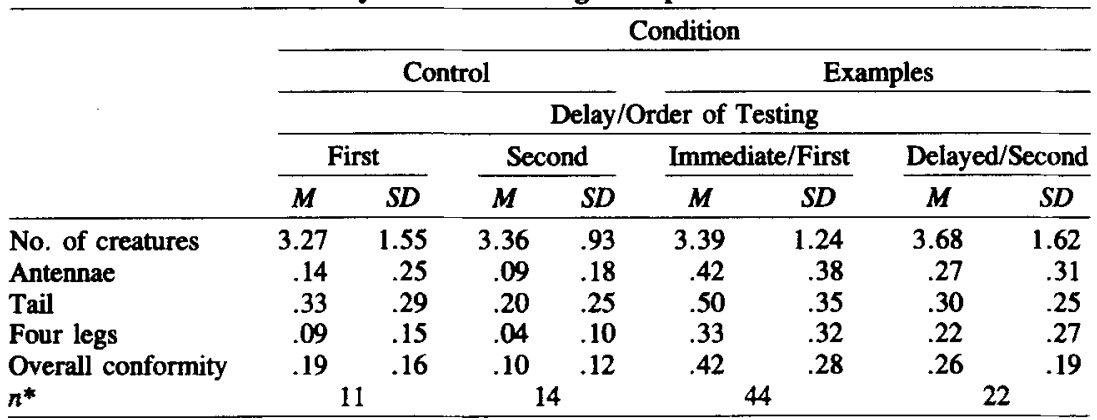

*Number of subjects for each group. 
in that direction. Longer delays may be needed to find a significantly diminished conformity effect.

\section{EXPERIMENT 3}

The constraining effects of seeing three similar examples prior to the idea-generation task were shown in both Experiments 1 and 2. A reasonable interpretation of the conformity effect is that it is unintentional, and based on the heightened accessibility of the properties of the recently experienced examples. Alternatively, however, it is possible that the results were obtained because of a subject bias effect. Specifically, it may be that the subjects believed that they were supposed to generate ideas similar to the provided examples. If this were the case, then the observed conformity effects could be avoided by instructing them to generate ideas as different from the examples as possible. Furthermore, if the subjects already believe that their ideas are supposed to conform to the examples, then instructions to conform should not increase the conformity effect.

In Experiment 3, subjects were given an idea-generation task with three similar examples, as in Experiments 1 and 2. Instructions for the different conditions were standard (exactly as in Experiments 1 and 2), diverge (ideas were to be as different from the examples as possible), or conform (ideas were to be as similar to the examples as possible). A no-examples control group was also included in the experimental design.

The memory hypothesis states that the observed conformity is caused by unintentional remembering of the examples, similar to output interference in memory tasks, an effect that cannot be overcome by instructions to generate ideas different from the examples. The subject bias hypothesis, however, predicts that instructions to generate altered designs will eliminate the conformity effect, and that instructions to conform should have no significant effect beyond the subject bias already inherent in the procedure.

\section{Method}

Subjects. The 80 participants were volunteers from introductory psychology classes who fulfilled part of a class requirement by participating in the experiment.
Design and Procedure. The design, procedure, and materials used in Experiment 3 were identical to those used in Experiment 1, with the following exceptions. Only one task, creature generation, was used in Experiment 3. Besides the standard examples and control conditions, as in Experiments 1 and 2, there were two additional treatments in Experiment 3. The examples/conform group was given the same example creatures as the examples group, but was also given the following instruction: "The examples you examined are some of the best ideas that people have had for this task. Although you should not copy any of these ideas exactly, it would be preferable if you could generate ideas like these." The examples/ diverge group was also shown the three examples, but they were told, "We have found that examples like those you examined restrict people's creativity. Try NOT to restrict your ideas. When the drawing task begins, please generate ideas as different as possible from the examples given."

\section{Results}

The results for Experiment 3 are shown in Table 4. For each dependent measure, a one-way ANOVA was computed to compare the four experimental treatment groups (control, standard examples, examples/conform, and examples/diverge). The number of creatures generated did not differ among the four treatment groups $[F(3,76)=$ $\left.1.05, M S_{c}=1.17\right]$, a finding consistent with the results of Experiments 1 and 2.

The results show that conformity had the same constraining effect as in the two previous experiments. Although the means did not significantly differ among the groups for the proportion of creatures generated with antennae $\left[F(3,76)=1.08, M S_{\mathrm{e}}=.05\right]$, there were significant differences for the proportion with a tail $[F(3,76)$ $\left.=9.27, M S_{\mathrm{e}}=.09\right]$ and the proportion with four legs $[F(3,76)=4.26]$

A significant effect could also be seen for the overall conformity of designs to examples $[F(3,76)=8.69$, $\left.M S_{\mathrm{e}}=.03\right]$. Planned orthogonal comparisons showed that the control group generated ideas with less overall conformity to the examples than did the three groups that were given examples $[t(76)=3.82]$. The control group was lower in overall conformity than the standard examples group, as in Experiments 1 and 2, but the effect was only marginal $[t(76)=1.56, .05<p<.10]$. The comparisons also showed that the control group had less overall conformity than the examples/diverge group $[t(76)=$ 2.89]. Thus, telling subjects to generate ideas that were

Table 4

Mean Number of Ideas Generated With Standard Deviations,

Proportions of Generated Creatures With Features of Examples, and

Overall Conformity as a Function of the Examples Given and Biasing Instructions in Experiment 3

\begin{tabular}{|c|c|c|c|c|c|c|c|c|}
\hline & \multirow{2}{*}{\multicolumn{2}{|c|}{ Control }} & \multicolumn{6}{|c|}{ Examples Condition Instructions } \\
\hline & & & \multicolumn{2}{|c|}{ Standard } & \multicolumn{2}{|c|}{ Diverge } & \multicolumn{2}{|c|}{ Conform } \\
\hline & $M$ & $S D$ & $M$ & $S D$ & $M$ & $S D$ & $M$ & $S D$ \\
\hline No. of creatures & 3.55 & 1.10 & 3.65 & .88 & 3.90 & 1.29 & 4.10 & 1.02 \\
\hline Antennae & .20 & .20 & .21 & .23 & .23 & .19 & .31 & .27 \\
\hline Tail & .16 & .21 & .34 & .30 & .53 & .38 & .62 & .27 \\
\hline Four legs & .13 & .20 & .20 & .23 & .22 & .26 & .39 & .26 \\
\hline Overall conformity & .16 & .13 & .25 & .17 & .33 & .20 & .44 & .20 \\
\hline$n^{*}$ & \multicolumn{2}{|c|}{20} & \multicolumn{2}{|c|}{20} & \multicolumn{2}{|c|}{20} & \multicolumn{2}{|c|}{20} \\
\hline
\end{tabular}

*Number of subjects for each group. 
very different from the examples did not eliminate the conformity effect. The standard examples group had less overall conformity than the examples/conform group $[t(76)=3.36]$; that is, instructions to conform increased conformity. Overall conformity for the standard examples group did not differ from that of the examples/diverge group $[t(76)=1.33]$, indicating again that instructions to diverge from the examples did not eliminate or diminish the conformity effect.

\section{Discussion}

In Experiment 3, as in Experiments 1 and 2, an effect of conformity was found; creative designs were more likely to contain features of the examples if examples had been shown to the subjects in advance. If the conformity effects in the three experiments had been caused by the subjects' making an implicit assumption that their generated ideas were supposed to conform to the examples, then asking them to conform to the examples should not have increased conformity, and asking them to create items that were very different from the examples should have decreased conformity. Both of these predictions were contradicted by the present results; instructions to conform increased conformity, and instructions to diverge from the examples did not decrease conformity. Therefore, the conformity associated with the examples conditions appears to be caused by unintentional memory of the examples, not by subjects' assumptions that they should try to conform to the examples.

The results are consistent with those obtained by Ward (in press). He found that subjects tended to create symmetric creatures with sense organs and appendages, even when they were instructed to use their wildest imagination and not to worry about creating an animal that would be believable to others. Thus, whether the task involves selfgenerated or experimenter-presented examples, conformity on properties of currently activated information is not simply a function of a perceived demand to create instances that have those properties.

\section{GENERAL DISCUSSION}

The three experiments support the hypothesis that conformity, induced by introducing examples, can constrain creative generation of imaginative ideas. Whether they were creating novel toys or creatures that could inhabit another planet, the subjects were affected by examples presented prior to the creative generation task. When examples were shown to them, subjects' subsequent creative ideas tended to conform to the examples. That is, compared with ideas generated by control groups, ideas generated by subjects who had seen examples contained more of the arbitrarily chosen features that were in the examples. This conformity effect persisted beyond a 23-min delay between viewing the examples and generating the novel ideas.

Conformity in the three experiments was not caused by the subjects' assuming that their ideas should be similar to the provided examples. When they were instructed to give ideas that resembled the examples in Experiment 3, conformity increased, indicating that the uninstructed subjects were not already deliberately attempting to conform. Furthermore, the conformity effect was not diminished when the subjects were explicitly instructed to generate ideas that were different from the provided examples. If the standard examples subjects had been assuming that ideas should conform to the examples, then instructions that told the subjects to try to diverge from the examples should have decreased or eliminated conformity effects. Because the instructions did not diminish the effect, the results imply that the observed conformity was unintentional, and not due to such assumptions.

The present results are consistent with previous observations that exemplar generation can be influenced by subjects' self-generated examples (Ward, in press). In the present case the examples were presented by the experimenter, and the findings clearly reveal the impact of recent experiences. In Ward's studies, when subjects began idea generation by spontaneously retrieving specific examples without being instructed to do so, their subsequent ideas tended to conform to their self-generated examples. The more general conclusion across these studies is that currently activated information can lead to conformity, regardless of the source of that activation.

The examples provided in the present experiments are analogous to inhibitory part-list cues in list-recall studies, which constrain retrieval efforts by inducing output interference (Rundus, 1973). Self-generated conformity effects, as Ward (in press) has described, would resemble the self-generated output interference caused by initial retrievals in free recall, which limit subsequent retrieval (Roediger, 1974). Whether items are initially retrieved from memory, or given as "cues" or "examples" by an experimenter, their effect is to inhibit retrieval of other related information.

The present results are consistent with the findings of Jansson and Smith (1991), who found that designs created by engineering students and professional designers conformed to examples given prior to their design work. Tasks in that study included designing spill-proof disposable cups and measuring cups for blind people. Features of the examples were more likely to appear in the designs of subjects who had seen an example, as compared with a control group that had seen no examples. This finding held even for unsuitable features of examples, as well as when subjects were explicitly told not to use a feature shown in the example. Furthermore, when an important feature was missing from an example, such as an overflow device in the measuring cup for the blind, it also tended to be omitted from the designs of the subjects who had seen the example.

In Jansson and Smith's (1991) study, the task of designing devices such as a spill-proof cup or measuring cup for the blind required some careful pragmatic considerations, and it posed problems that needed realistic solutions. The examples solved the problems even when the 
solutions provided by the examples were less than optimal. The conformity effects observed in that study, therefore, could have been caused by difficulty in thinking of alternate solutions once subjects had seen one solution. The present study extends Jansson and Smith's findings by showing that example-induced conformity occurs even when ideas need not solve any problems or meet any pragmatic constraints, and when the properties of the examples do not provide obvious answers to well-defined problems. Furthermore, it was not known whether or not Jansson and Smith's subjects tacitly assumed that they were expected to create designs similar to the examples. The results of Experiment 3 in the present study indicate that the conformity observed in creative generation tasks is not caused by such assumptions.

The present study is concerned with creative cognition, the cognitive underpinnings of creative thinking (Finke et al., 1992). In this case, we have examined some limiting effects of recent memories on creative idea generation. We do not wish to imply that conforming to the attributes of earlier experiences must always be undesirable. Obviously, learning and benefiting from prior experience is one of the most important human adaptive traits, and can be important even in creative problem solving (e.g., Weisberg \& Alba, 1981). In creative idea generation, however, novelty is one of the most important characteristics of ideas, perhaps because creative ideas are most needed when known, accepted ideas are no longer adequate. Constraints, particularly unintentional ones, may be especially limiting to the discovery of novel ideas.

\section{REFERENCES}

Barsalou, L. W. (1987). The instability of graded structure: Implications for the nature of concepts. In U. Neisser (Ed.), Concepts and conceptual development: Ecological and intellectual factors in categorization (pp. 101-140). Cambridge: Cambridge University Press.
Bransford, J. D., Barclay, J. R., \& Franks, J. J. (1972). Sentence memory: A constructive versus interpretive approach. Cognitive Psychology, 3, 193-209.

Finke, R. A., WARD, T. B., SMITh, S. M. (1992). Creative cognition: Theory, research, and applications. Cambridge, MA: MIT Press.

Gick, M. L., \& Holyoak, K. J. (1980). Analogical problem solving. Cognitive Psychology, 12, 306-355.

Jansson, D. G., \& SMTth, S. M. (1991). Design fixation. Design Studies, 12, 3-11.

Luchins, A. S., \& Luchins, E. H. (1959). Rigidity of behavior. Eugene, OR: University of Oregon Press.

Nickerson, R. S. (1984). Retrieval inhibition from part-set cuing: A persisting enigma in memory research. Memory \& Cognition, 12, $531-552$.

RaAijmakers, J. G. W., \& Shiffrin, R. M. (1981). Search of associative memory. Psychological Review, 88, 93-134.

RoEDiger, H. L., III (1974). Inhibiting effects of recall. Memory \& Cognition, 2, 261-269.

Roediger, H. L., III, \& NeEly, J. H. (1982). Retrieval blocks in episodic and semantic memory. Canadian Journal of Psychology, 36, 213-242.

Ross, B. H., Ryan, W. J., \& Tenpenny, P. L. (1989). The access of relevant information for solving problems. Memory \& Cognition, 17, 639-651.

Rundus, D. (1973). Negative effects of using list items as recall cues. Journal of Verbal Learning \& Verbal Behavior, 12, 43-50.

SLAMECKA, N. J. (1968). An examination of trace storage in free recall. Journal of Experimental Psychology, 76, 504-513.

SMITH, S. M., \& BLANKENSHIP, S. E. (1989). Incubation effects. Bulletin of the Psychonomic Society, 27, 311-314.

SmIth, S. M., \& BlankENSHIP, S. E. (1991). Incubation and the persistence of fixation in problem solving. American Journal of Psychology, 104, 61-87.

WARD, T. B. (in press). Structured imagination: The role of category structure in exemplar generation. Cognitive Psychology.

WeisBerg, R. W., \& AlBA, J. W. (1981). An examination of the alleged role of "fixation" in the solution of several "insight" problems. Journal of Experimental Psychology: General, 110, 169-192.

(Manuscript received December 31, 1992; revision accepted for publication April 26, 1993.) 